\title{
Functional analysis of a novel C-glycosyltransferase in the orchid Dendrobium catenatum
}

\author{
Zhiyao Ren ${ }^{1}$, Xiaoyu $\mathrm{Ji}^{2}$, Zhenbin Jiao ${ }^{3,4}$, Yingyi Luo ${ }^{6}$, Guo-Qiang Zhang ${ }^{5}$, Shengchang Tao ${ }^{1,7}$, Zhouxi Lei ${ }^{1}$, \\ Jing Zhang ${ }^{1}$, Yuchen Wang ${ }^{1}$, Zhong-Jian Liu' ${ }^{8,9}$ and Gang Wei (1)
}

\begin{abstract}
Flavonoids, which are a diverse class of phytonutrients, are used by organisms to respond to nearly all abiotic stresses and are beneficial for human health. Glycosyltransferase, used during the last step of flavonoid biosynthesis, is important in flavonoid enrichment. However, little is known about glycosyltransferase in the orchid Dendrobium catenatum (D. officinale). In this study, we isolated a novel C-glycosyltransferase (designated DcaCGT) from the orchid D. catenatum by identifying and analyzing 82 putative genes in the GT1 family. DcaCGT could specifically catalyze not only di-C-glycosylation but also O-glycosylation. Apart from the normal function of catalyzing 2-hydroxynaringenin and phloretin to the respective di-C-glycosides, DcaCGT also catalyzes apigenin to cosmosiin. Targeted metabolic profiling of the substrates (2-hydroxynaringenin, phloretin, and apigenin) and products (vitexin, isovitexin, vicenin-2, nothofagin, 3',5'-di-C-glucosylphloretin, and cosmosiin) in different tissues showed that vicenin-2 was the most abundant product of this novel enzyme. Cosmosiin was detected in flowers and flower buds. We also established that DcaCGT functions expanded throughout the evolution of $D$. catenatum. Residual OGT activity may help $D$. catenatum resist drought stress. Our study illustrates the function, origin, and differentiation of DcaCGT and provides insights into glycosylation and molecular propagation processes, which can be used to improve the production of flavonoids by the cultivated medicinal plant D. catenatum.
\end{abstract}

\section{Introduction}

Flavonoids, a class of valuable secondary metabolites, are widely distributed in plants. More than 10,000 flavonoids and their derivatives have been identified to date ${ }^{1}$. Flavonoid glycosides are often modified by flavonoid $O$ and $C$-glycosylation, which change the biological activities of these compounds. In plants, $C$-glycosylflavones protect against UV-B irradiation ${ }^{2}$, exert antimicrobial effects ${ }^{3}$, and are involved in plant allelopathy ${ }^{4}$ and copigmentation $^{5}$. C-glycosylflavones are critical in plant physiology and are beneficial for human health. For example, vicenin2 is an effective flavone that shows anti-inflammatory ${ }^{6}$, antiglycating ${ }^{7}$, antispasmodic ${ }^{8}$, antiseptic $^{9}$, antiplatelet,

\footnotetext{
Correspondence: Zhong-Jian Liu (zjliu@fafu.edu.cn) or

Gang Wei (weigang021@outlook.com)

${ }^{1}$ School of Pharmaceutical Sciences, Guangzhou University of Chinese Medicine, Guangzhou 510006, China

${ }^{2}$ Shantou University Medical College, Shantou 515041, China

Full list of author information is available at the end of the article

These authors contributed equally: Zhiyao Ren, Xiaoyu Ji
}

antithrombotic $^{10}$, and anticancer ${ }^{11,12}$ activity. Because flavonoids show such high biological activity, determining the pathways involved in the biosynthesis of flavonoid glycosides is important.

Glycosylation has an important role in modifying secondary metabolites, maintaining metabolic homeostasis, and detoxifying xenobiotics. These processes are mediated by a set of glycosyltransferases (GTs, EC 2.4.x.y). These enzymes are responsible for catalyzing glycosylation and are classified into 109 families according to the similarity of their amino-acid sequences in the Carbohydrate-Active enZYmes database (http://www. cazy.org). Family-1 glycosyltransferases are the largest multigene GT family, and their glycosylated products participate in the regulation of plant growth and development, such as hormone homeostasis ${ }^{13}$, pollination ${ }^{14}$, and interactions with the environment, such as detoxification of xenobiotics ${ }^{15}$ as well as UV tolerance ${ }^{16}$. GT1s are also called UDP-glycosyltransferases because they use 
uridine 5'-diphosphate as a sugar donor. UGTs are characterized by a highly conserved motif of a 44-aminoacid C-terminal consensus sequence called the plant secondary product glycosyltransferase (PSPG) box. Because the GT1 family has such a crucial role in the synthesis of plant secondary metabolites, members of this family have been identified mostly in model plants, crops, and fruits such as Arabidopsis (Arabidopsis thaliana) ${ }^{17}$, maize (Zea mays) ${ }^{18}$, wheat (Triticum aestivum) ${ }^{19}$, soybean

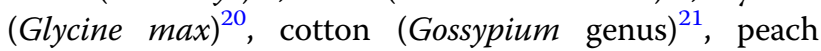

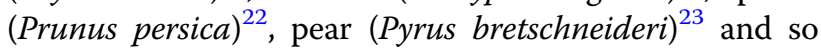
on. However, little is known about the GT1 family in medicinal plants.

Dendrobium catenatum (D. officinale), a perennial herb in the Orchidaceae, is rich in flavonoid glycosides ${ }^{24}$ and is mainly distributed in Southeast, South, and Southwest China $^{25}$. In the wild, D. catenatum largely grows year round on cliffs that receive abundant sunlight and scant water $^{26}$. Owing to its high medicinal value, $D$. catenatum has been widely used in traditional Chinese medicine ${ }^{27}$. According to Chinese medical theory, the stems of $D$. catenatum can nourish yin, enhance gastric motility, and increase body fluids regulating immunity ${ }^{28}$. Modern pharmacological studies have shown that the active ingredients of $D$. catenatum include polysaccharides ${ }^{29}$, flavonoids ${ }^{26}$, and bibenzyls ${ }^{30}$, which promote salivation and relieve the symptoms of diabetes ${ }^{31,32}$.

Glycosylation is the final step in the synthesis of flavonoid glycosides. Using oxygen, $O$-glycosyltransferases link the $\mathrm{O}$-glycosylflavone sugar moiety to the flavonoid skeleton, whereas the glucose moiety of $C$-glycosylflavones is directly bound to the flavone backbone. $C$-glycosylflavone biosynthesis is catalyzed by $C$-glycosyltransferases (CGTs). In 2009, CGTs were first reported in rice $\left(\right.$ OsCGT) ${ }^{33}$, followed by maize (UGT708A6) ${ }^{34}$, buckwheat $(\mathrm{FeCGTa}, \mathrm{FeCGTb})^{35}$, and soybean (UGT708D1) ${ }^{36}$. Most $C$-glycosylflavones are synthesized via glucosylation of the substrate 2-hydroxyflavanone, followed by dehydrasemediated dehydration. In 2014, another GtUF6CGTmediated $\mathrm{C}$-glycosylation pathway was identified in Gentiana triflora, showing that the sugar moiety can be directly catalyzed to the flavone skeleton ${ }^{37}$. In 2017, the first CGT catalyzing the glycosylation of di- $C$-glycosides was found in citrus fruits (UGT708G1, UGT708G2) ${ }^{38}$. In 2019 , the first flavone $8-C$-glycosyltransferase was discovered in Trollius chinensis (TcCGT) ${ }^{39}$.

$\mathrm{Di}-\mathrm{C}$-glycosylflavones are the main flavonoids in $D$. catenatum $^{40}$. However, the amounts of secondary metabolites in D. catenatum vary regionally. Higher levels of di- $C$-glycosylflavones may help this plant adapt to a harsh growing environment and affect the levels of medically important ingredients. Although the last step in the formation of $C$-glycosylflavones is well characterized in plants, the gene involved in the biosynthesis of di-C- glycosylflavones in D. catenatum has not been identified. In this study, we performed a genome-wide analysis and functional verification of CGT genes in D. catenatum. The levels of DcaCGT-related flavonoids were evaluated by ultra-high-performance liquid chromatography coupled with triple-quadrupole mass spectrometry. The haplotype distribution of DcaCGT was assessed in 274 samples acquired from 34 populations. Our study provides the biosynthetic pathways of di-C/O-glucosylflavones in $D$. catenatum, and performs a molecular phylogenetic analysis to offer insight into the evolution of the functional gene.

\section{Results}

\section{Identification of UGT genes in D. catenatum}

A BLASTP search of the D. catenatum genome (GenBank accession number: PRJNA262478) ${ }^{29}$ was performed using the UGT-conserved PSPG box sequence as a query. A total of $82 D$. catenatum UGTs $\left(E\right.$ value $\left.<10^{-5}\right)$ having lengths of 297-609 amino acids (with an average length of 471 amino acids) were identified. A phylogenetic tree of $D$. catenatum UGT genes was constructed by aligning the full-length amino-acid sequences of $D$. catenatum UGTs with functionally characterized plant UGTs, including Arabidopsis and maize UGTs (Fig. 1). These D. catenatum UGTs were phylogenetically divided into 16 groups, including 12 groups (A-G, I-M) that were identified in Arabidopsis ${ }^{17}, 3$ groups $(\mathrm{O}-\mathrm{Q})$ that were identified in maize ${ }^{18}$ and 1 group (R) that was found in seed plants ${ }^{41}$. Among the 82 listed UGTs, few were functionally characterized. The predicted molecular weight ranged from 33 to $68 \mathrm{kDa}$. The isoelectric point ranged from 4.79 to 8.9 (Table S1). Based on homology analysis, CGT gene candidates were selected from the 82 putative UGTs.

\section{Chromosomal distribution of $D$. catenatum UGT genes}

The genomic distribution of each $U G T$ is shown in Fig. 2 to provide an overview of the location of $D$. catenatum UGT genes. The D. catenatum genome contained 19 chromosomes, but the 82 UGTs were distributed across only 15 chromosomes (Fig. 2). The four UGTs on the low right side of the figure are located on scaffolds and include Dca026746, Dca027036, Dca028143, and Dca028752. Different members of the UGT family were observed on each chromosome. Twenty-five UGTs were located on the longest chromosome, 01 , followed by 11 members each on chromosomes 02 and 03 and 8 UGTs on chromosome 04. In addition, 1-3 UGTs were distributed on the other 11 chromosomes. Members of the same group were observed on several different chromosomes. UGTs belonging to the same group on the same chromosome tended to cluster together, whereas others were clustered separately. Groups D and E, containing the highest and second highest number of genes, respectively, were 


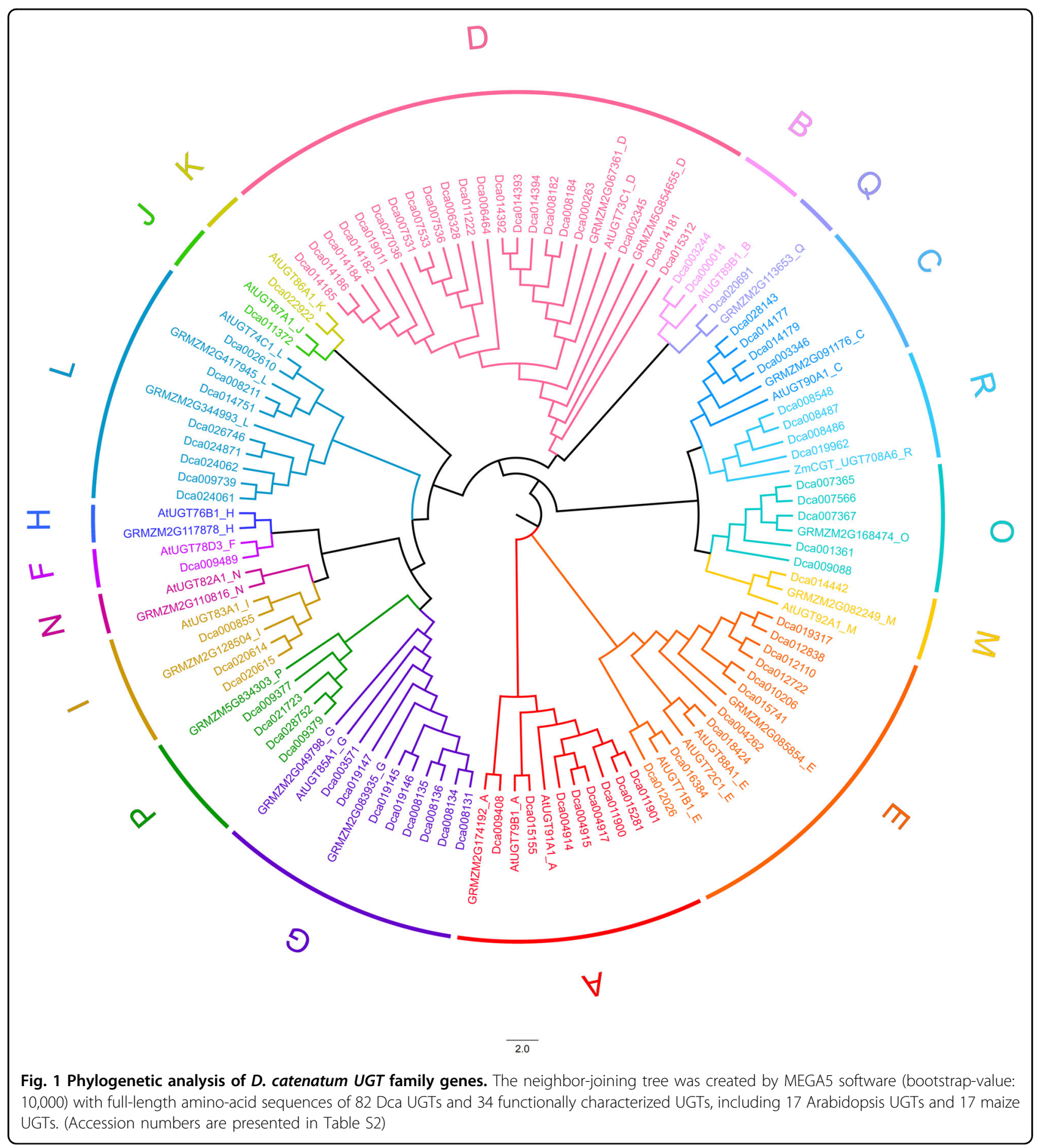

randomly distributed across five different chromosomes (Table S1).

\section{Sequence analysis of $D$. catenatum UGT genes}

Next, we analyzed exon-intron organization to investigate the evolutionary relationships within the $D$. catenatum UGT gene family. Among the 82 UGTs, 43 contained no introns, and 30 contained one intron in each gene. For the remaining nine $U G T s$, seven contained two introns, and only one $U G T$ each contained three and five introns. UGTs without introns occurred more commonly than those with introns. For the UGT groups, $100 \%$ intron loss was observed in genes of groups $\mathrm{B}, \mathrm{C}$, and $\mathrm{Q}$, followed by $76 \%$ of genes in group $\mathrm{D}$ and $60 \%$ of genes in group $\mathrm{M}$. Conversely, all genes in groups F, G, H, I, and J contained introns. A total of seven (87\%) UGTs in group G 


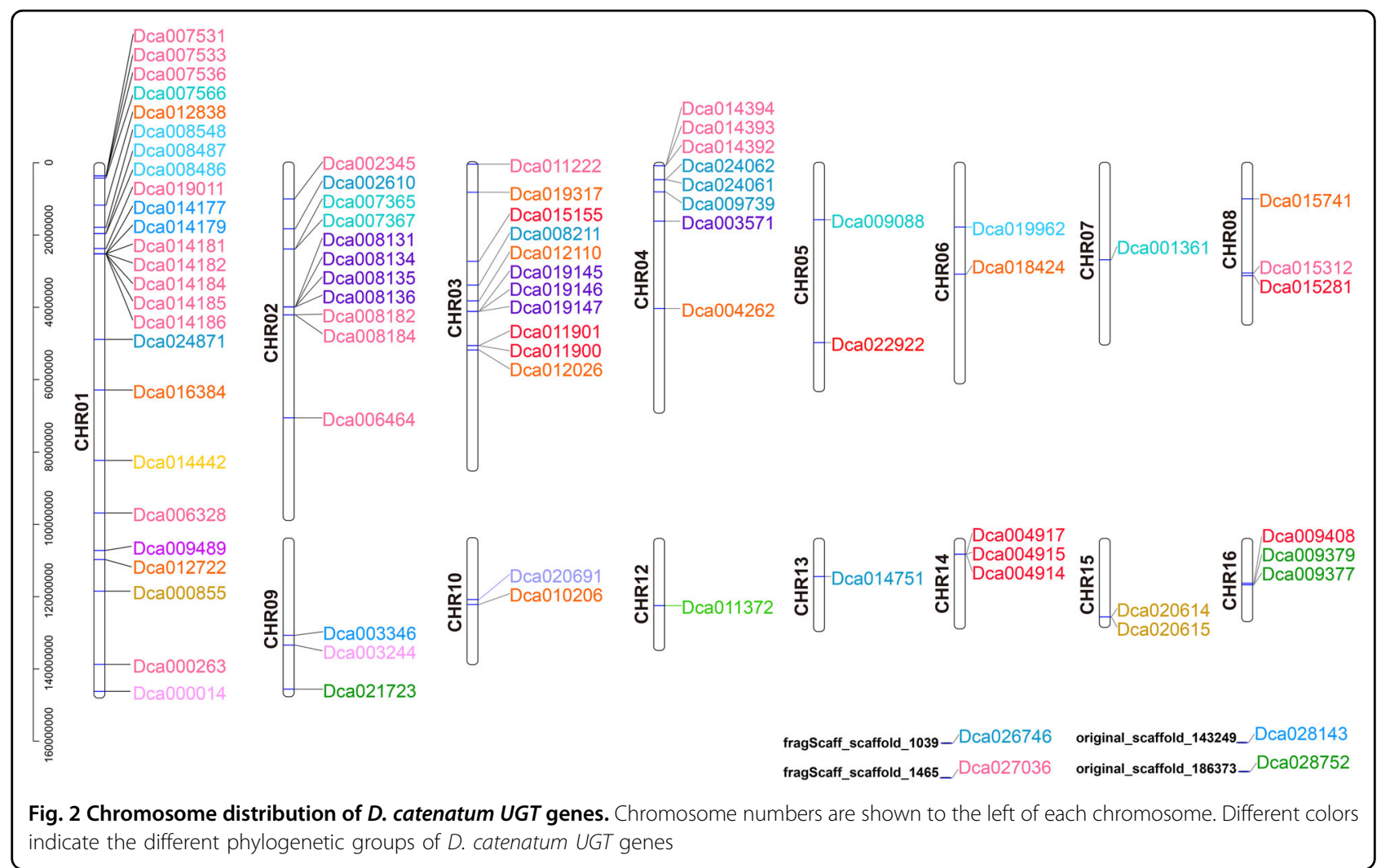

contained one intron. These positions of intron insertion were determined to be highly conserved after mapping the introns via alignment of amino-acid sequences. Introninsertion positions varied in groups $\mathrm{A}, \mathrm{E}, \mathrm{O}$, and $\mathrm{R}$. Among the 52 total introns detected in $D$. catenatum UGT sequences, 48, 2, and 2 were in phases 0,1 , and 2 , respectively. In addition, $92 \%$ of introns were in phase 0 , which is far greater than the number in phases 1 and 2 . Based on the information presented above, introns in $D$. catenatum family-1 UDP-glycosyltransferase clusters were deemed to not be completely conserved. In addition, an NCBI CD search showed that most of the 82 UGTs were GTB-type glycosyltransferases (Fig. 3b).

A schematic representation of the structure of all $D$. catenatum UGT proteins was constructed from the results of MEME motif analysis. As shown in Fig. 3a, motifs 1,2 , and 5 were widely distributed in every gene. Motif 1 was the location of the PSPG box, whereas the protein architecture of motifs 2 and 5 was speculated to be conserved in $D$. catenatum UGTs. Members within the same groups were usually found to share similar structures. For example, all members in group $\mathrm{O}$, three of four genes in group $R$ and 7 of 10 genes in group $E$ showed the absence of motif 9 . Three-fifths of the UGTs in group O showed the loss of motif 8 . Together with the results of the phylogenetic analysis, this similarity in motif arrangements among $D$. catenatum UGT proteins within subgroups indicates that the subfamily classification of UGTs may be based on partially conserved protein domains.

\section{Gene expression analysis of different organs of $D$. catenatum}

The transcriptome revealed 82 UGTs with varied expression patterns across 10 plant tissues (Fig. 4), including the bud, gynandrium, labellum, sepal, root tip, day root (root1), leaf, stem, pollen, and night root (root2) (GenBank accession number: PRJNA348403) ${ }^{42}$. Sixty-nine UGT genes were expressed in at least two organs, whereas transcription of 13 UGTs was not observed. For the unexpressed UGTs, three genes were located in groups $\mathrm{D}$ and $L$, two genes were located in group $E$, and the other six genes were distributed among groups A, C, G, I, and P. We examined the possible expression pattern of UGTs in different tissues and found that 58 UGTs were expressed in the bud and sepal, which were the organs showing the highest number of expressed UGTs. The lowest number of UGTs (49) was found in pollen tissue. The number of UGTs expressed in other organs, such as the gynandrium, labellum, root tip, day root, leaf, stem, and night root, ranged between 49 and 58 . We also observed that unexpressed genes in these tissues were mostly distributed in groups $\mathrm{D}, \mathrm{G}, \mathrm{L}$, and $\mathrm{E}$. In contrast to 35 UGTs, which were expressed in all nine plant tissue samples, nine UGTs were 




Fig. 3 Gene structure and architecture of conserved protein motifs in UGT family genes of $\boldsymbol{D}$. catenatum. a Motif composition of UGTs; colored boxes indicate 10 different motifs. b Exon-intron structure and NCBI CD search results of UGT genes. Colored boxes indicate exons; black lines indicate introns; and numbers indicate the corresponding intron phases

expressed in all but one organ sample. Dca027036 and Dca015281 expression was not detected in the gynandrium, that of Dca015155 and Dca012026 was not detected in leaves, that of Dca009379, Dca014392, Dca012722, and Dca002610 was not detected in pollen, and that of Dca008134 was not detected in the night root. 


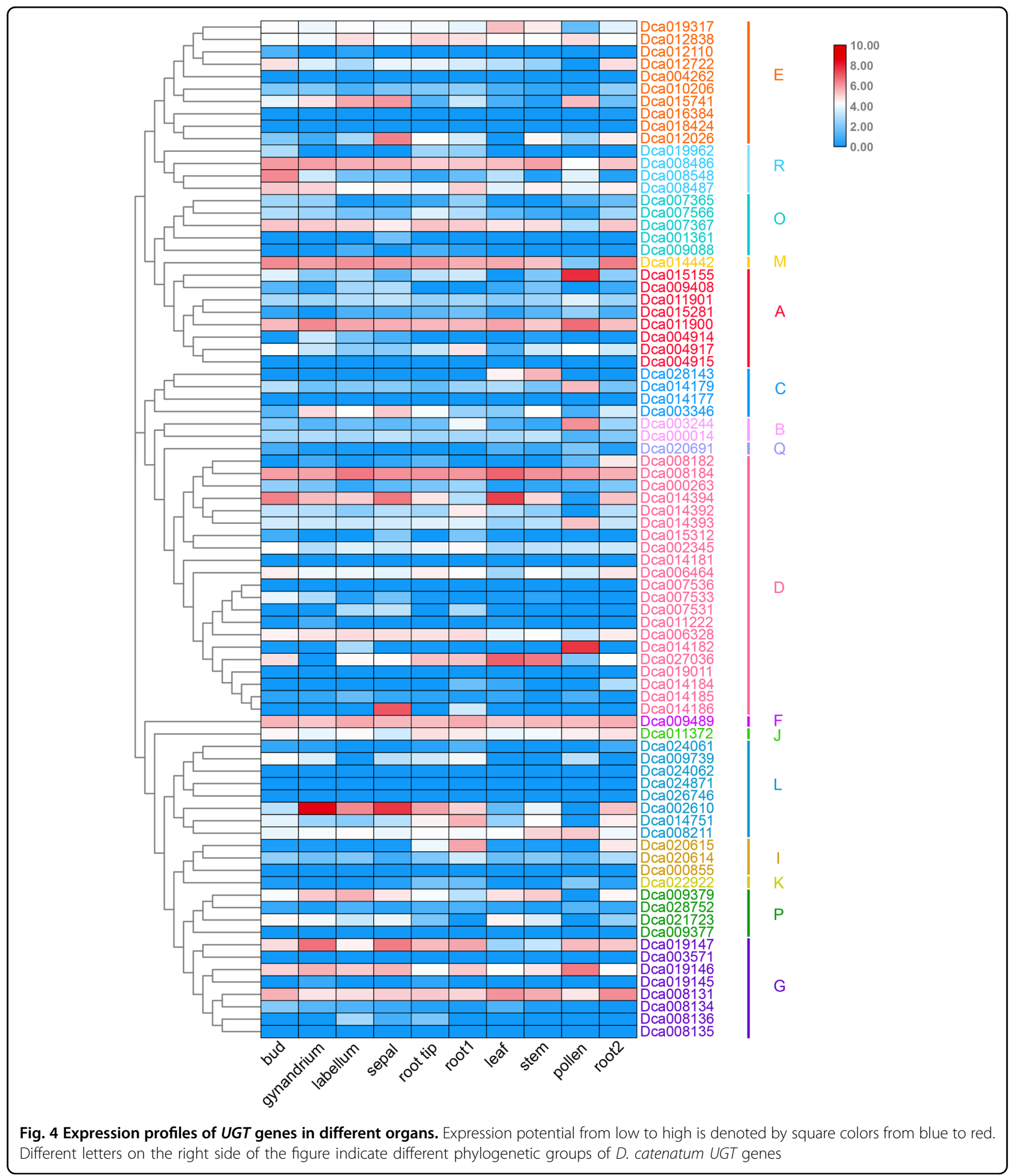

These results suggest that high transcription levels of a specific $U G T$ may lead to increased levels of related components, whereas unexpressed UGTs in different organs may result in the absence of certain components in these organs.
Properties of recombinant $D$. catenatum CGT expressed in $E$. coli and in vitro enzymatic activity assays

To determine the functions of DcaCGT, full-length cDNA was obtained from $D$. catenatum. The CDS region was cloned into a pET32a $(+)$ vector and transformed into 


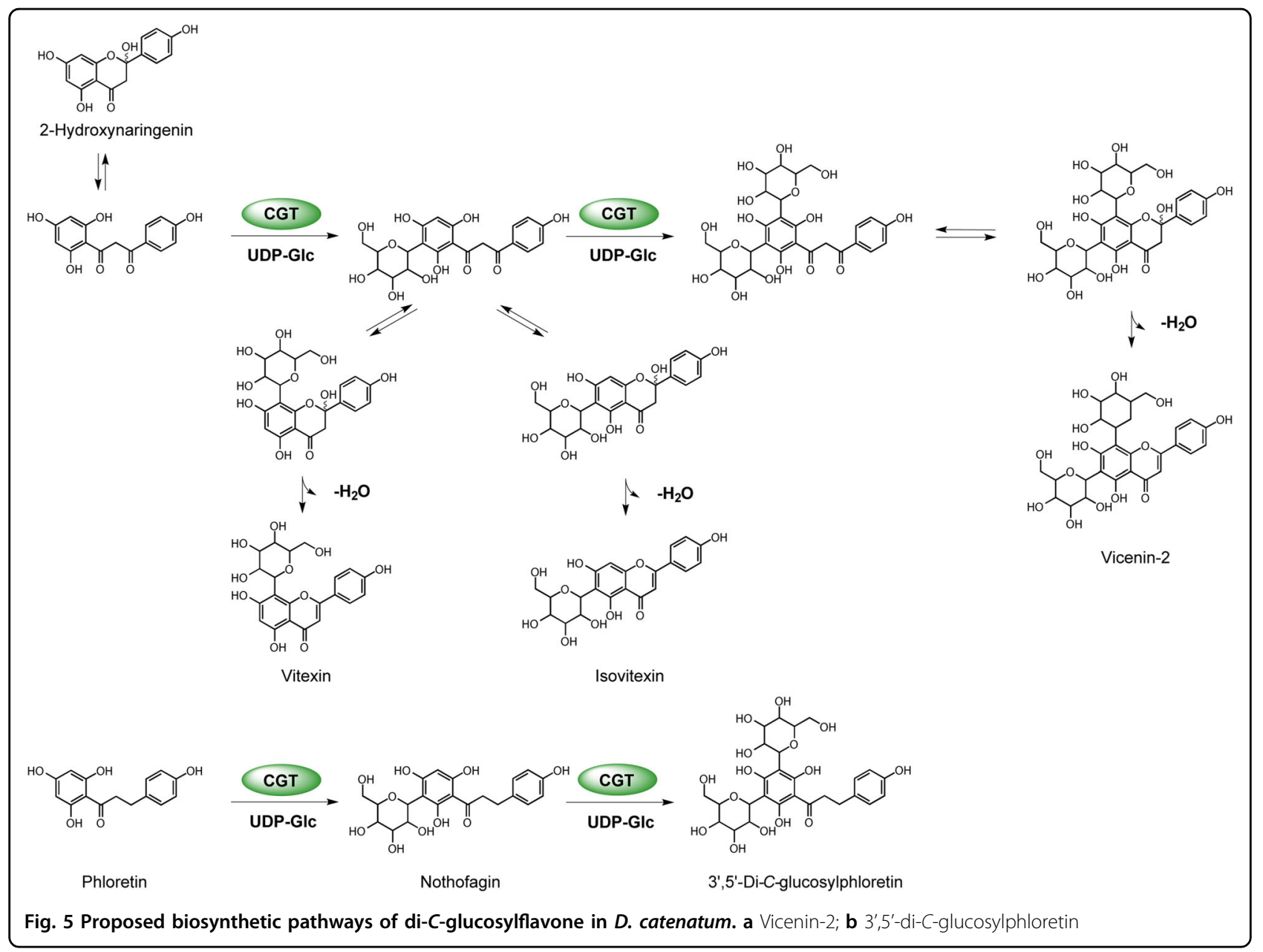

Escherichia coli Rosetta (DE3) to investigate the catalytic activity of the enzyme encoded by the isolated putative DcaCGT gene (GenBank accession number: MT452646). The expressed recombinant proteins contained a fused His6 tag and showed a molecular weight of $66.1 \mathrm{kDa}$ when analyzed via SDS-PAGE (Fig. S1). Enzymatic activity assays were conducted using three flavanone substrates: 2-hydroxynaringenin, phloretin, and apigenin. Glycoside products were analyzed by liquid chromatography-mass spectrometry (LC-MS).

The resulting recombinant protein DcaCGT displayed $C$-glucosylation activity against 2-hydroxynaringenin, indicating that it was a CGT derived from $D$. catenatum plants. The typical reaction pattern catalyzed by DcaCGT is shown in Fig. 5. When 2-hydroxynaringenin was used as the substrate, it was converted into 6-Cglucosyl-2-hydroxynaringenin at $\mathrm{m} / \mathrm{z} 449$ (peak 5) and 6,8-di-C-glucosyl-2-hydroxynaringenin at $\mathrm{m} / \mathrm{z} 611$ (peak 6); these were the main products following the enzymatic reaction (Fig. 6). After treatment with $\mathrm{HCl}$, these compounds were dehydrated. Two products of dehydrated derivatives (peaks 2 and 3) showed $C$-glycoside moiety fragment ions of $[\mathrm{M}-\mathrm{H}-90]^{-}$and $[\mathrm{M}-\mathrm{H}-$ $120]^{-43}$, which corresponded to vitexin $(\mathrm{m} / \mathrm{z} 431)$ and isovitexin $(\mathrm{m} / \mathrm{z} 431)$, respectively. Vitexin and isovitexin levels decreased as the reaction continued, whereas the level of vicenin-2 (m/z 593) increased (Fig. S2). All the dehydrated products showed the same retention time and fragmentation pattern as those of the respective standards ${ }^{44}$. Moreover, two products were observed when phloretin was used as the substrate. The $\mathrm{MS}^{2}$ spectrum of peak 8 at m/z $345[\mathrm{M}-\mathrm{H}-90]^{-}$and 315 [M$\mathrm{H}-120]^{-}$showed the presence of nothofagin ${ }^{45}$. Peak 9 showed a pseudomolecular ion at $\mathrm{m} / \mathrm{z} 597$. The fragments at $\mathrm{m} / \mathrm{z} 477$ [M-H-120] ${ }^{-}, 417$ [M-H-90-90] $]^{-}$, and 357 [M-H-120-120] ${ }^{-}$suggested that most of these were gained from the loss of sugar residues (Fig. S3). Based on ESI-MS data, this product was identified as $3^{\prime}, 5^{\prime}-\mathrm{di}-\mathrm{C}$ glucosylphloretin ${ }^{46}$. These results indicate that $D c a C G T$ encodes a glycosyltransferase that catalyzes the conversion of flavanones to flavonoid $\mathrm{di}-\mathrm{C}$-glucoside via flavonoid mono- $C$-glucoside intermediates. 


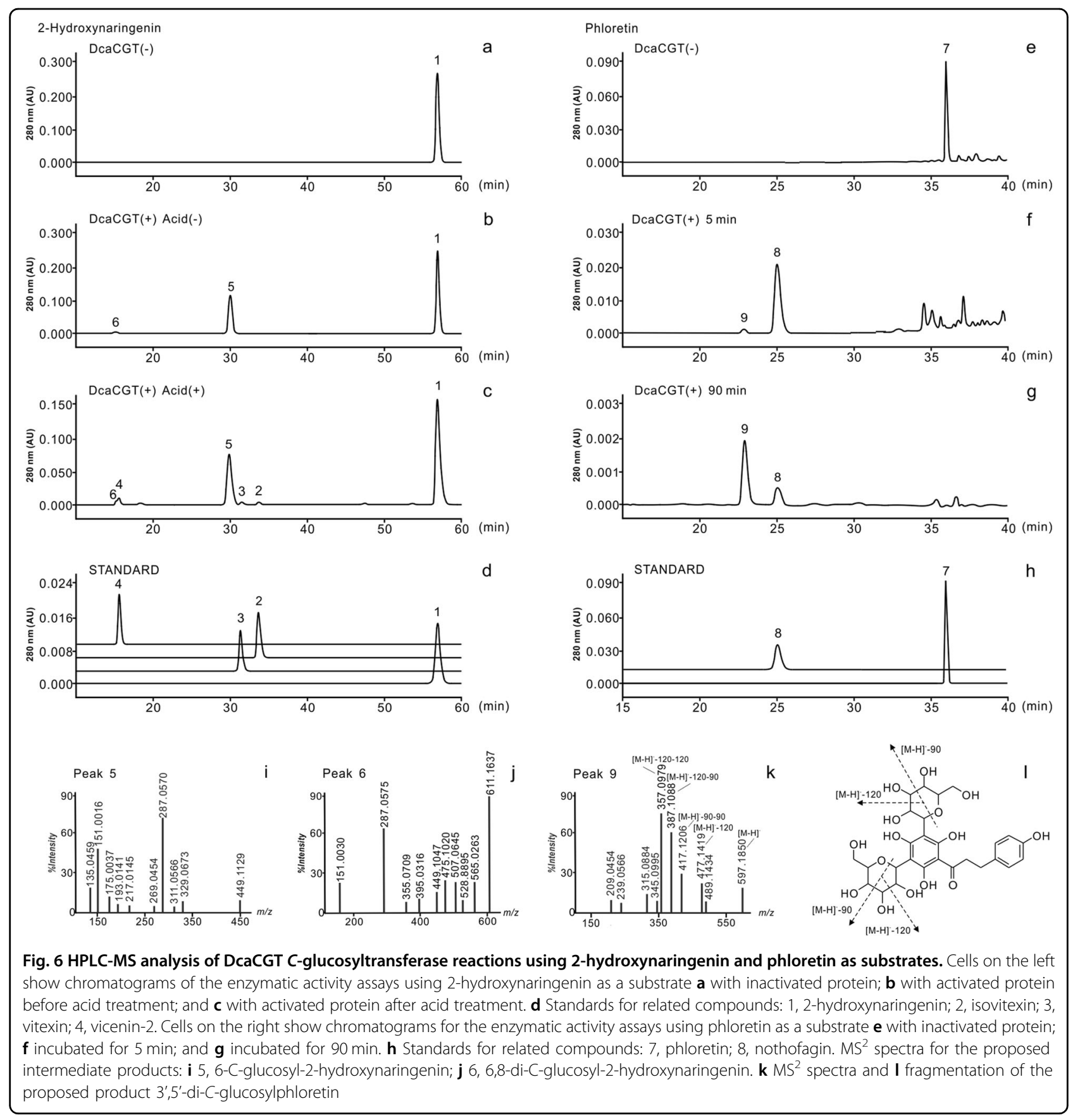

In addition, when we used apigenin as a substrate, a new product was detected by LC-MS (Fig. 7). MS analysis showed a precursor at $\mathrm{m} / \mathrm{z} 431$ (Fig. S4). The fragments at $\mathrm{m} / \mathrm{z}$ [M-H-162] $]^{-}$indicated apigenin bonding to glucose via $O$-linkage ${ }^{47}$. The new product exhibited the same retention time and mass fragmentation pattern as those of the authentic standard for cosmosiin. Hence, these results support those obtained using bioconversion assays and indicate that DcaCGT can catalyze substrates to not only di-C-glycosylflavone but also $O$-glycosylflavone.

\section{Flavonoids of $D$. catenatum}

The biosynthesis of flavones (vitexin, isovitexin, vicenin2, nothofagin, 3',5'-di-C-glucosylphloretin, and cosmosiin) from flavanones (2-hydroxynaringenin, phloretin, and apigenin) is catalyzed by UDP-glycosyltransferases. To explore the distribution of UGT-related flavanones and flavone glycosides accumulated in $D$. catenatum, we prepared methanolic extracts from flower buds, flowers, leaves, stems, and roots for UPLC-MS analysis. The chromatogram and mass spectrometry conditions of the 


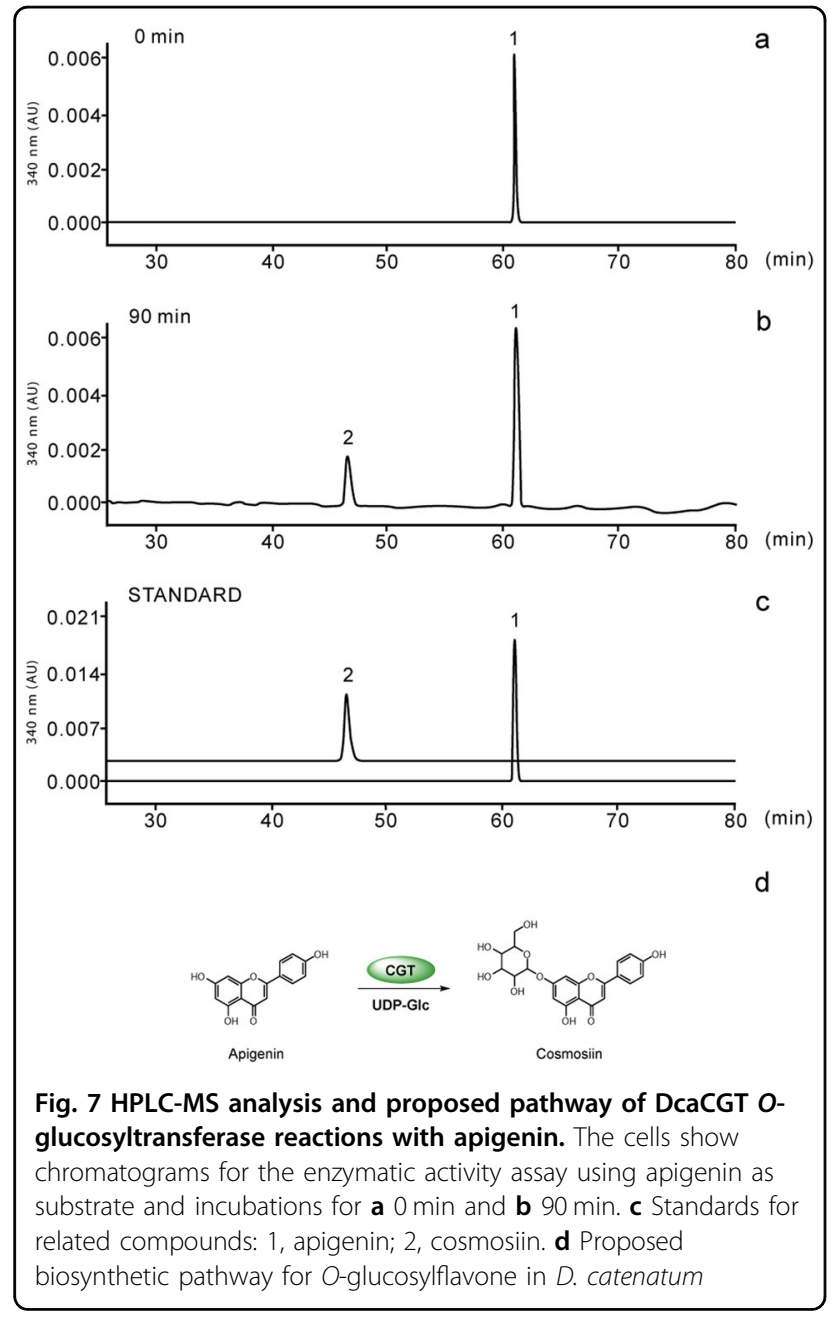

eight investigated compounds as well as the internal standard are shown in Fig. S5 and Table S4. Among the nine targeted flavonoids, eight compounds (except 3',5'di- $C$-glucosylphloretin) were found in the different organs. The compositions of these compounds varied among the organs (Fig. S6). C-glycosylflavone accumulation was significantly higher than that of $O$-glycosylflavones in the plant tissues examined in this study. Vicenin-2 and its intermediate products were more abundant than nothofagin and its intermediate products. 2-Hydroxynaringenin, phloretin, and apigenin were mostly detected in the roots, stems, and leaves, while vicenin-2, vitexin, isovitexin, nothofagin, and cosmosiin showed higher levels in flowers and flower buds. Flower buds showed the greatest variety of flavone glycosides and included vitexin, isovitexin, and vicenin-2, whereas roots had the lowest levels of vicenin-2 and intermediate products. These results, together with those of transcriptomic analysis, indicate that DcaUGT may mainly be used to biosynthesize vicenin-2 and that higher levels of vicenin-2 may be related to higher DcaUGT transcript levels in $D$. catenatum. Other substrates and products, such as apigenin and cosmosiin, were not detected simultaneously in the same organ. This may be related to different periods of gene expression and harvest times (Fig. 8 and Table 1).

\section{Molecular phylogenetic analysis of $D$. catenatum CGTs}

A neighbor-joining tree was constructed based on the deduced amino-acid sequences of the seven clusters (Fig. 9) and included flavone 7-OH glycosylation (cluster 1), flavonoid glycoside sugar-O-sugar links (cluster 2), 3-OH glycosylation (cluster 3 ), 5-OH glycosylation (cluster 4), isoflavone 7-OH glycosylation (cluster 5), plant CGTs (cluster 6), and bacterial CGTs (cluster 7). Detailed information on these UGTs is summarized in Table S5. DcaCGT is listed in cluster 6 with other plant CGTs, indicating that they may have evolved from the same ancestral gene. DcaCGT and ZmCGT were grouped into a clade, suggesting that they may have similar active sites used to catalyze flavonoid $\mathrm{C}$-glycosides and $\mathrm{O}$-glycosides simultaneously. Alignments of the amino-acid sequences of DcaCGT and other plant $C$-glycosyltransferases are shown in Fig. S7.

\section{Multiple sequence alignment analysis of DcaCGT from $D$. catenatum collected from different geographical locales}

To examine the evolutionary origin of DcaCGT, fulllength DcaCGT was extracted and analyzed from 279 accessions of Dendrobium, including resequencing data from $274 D$. catenatum samples obtained from 34 populations and data from five samples of $D$. huoshanense obtained from a single population. As many as 132 haplotypes were observed among the aligned sequence data from the $274 D$. catenatum samples. The $D$. huoshanense population was chosen as the root. Phylogenetic analysis indicated that DcaCGT haplotypes were grouped mainly into three clusters: the ancestral Cluster_a and evolutionary clade Clusters_b and _c, with 100 and 9.8\% bootstrap support, respectively (Fig. 10a). In addition, the 274 samples were unevenly distributed among the three clades, with Cluster_a containing 46 samples (32 haplotypes), Cluster_b containing 72 samples (36 haplotypes), and Cluster_c containing 156 samples (64 haplotypes). A comparison of nucleotide diversity among these three clusters indicated that Cluster_a $(\theta=0.00752 ; \pi=$ $0.00461)$ exhibited higher diversity than Cluster_b $(\theta=$ $0.00576 ; \pi=0.00244)$ and Cluster_c $(\theta=0.00705 ; \pi=$ 0.00287). Negative Tajima's $D$ values were observed among these clades. However, significant negative Tajima's $D$ values were only observed in the evolutionary clades for Cluster_b and Cluster_c. DcaCGT, the CGT identified in this study, was grouped in Cluster_c. These results suggest that natural selection acted on the evolutionary clades. Significant differentiation was found among Cluster_a, Cluster_b, and Cluster_c because 


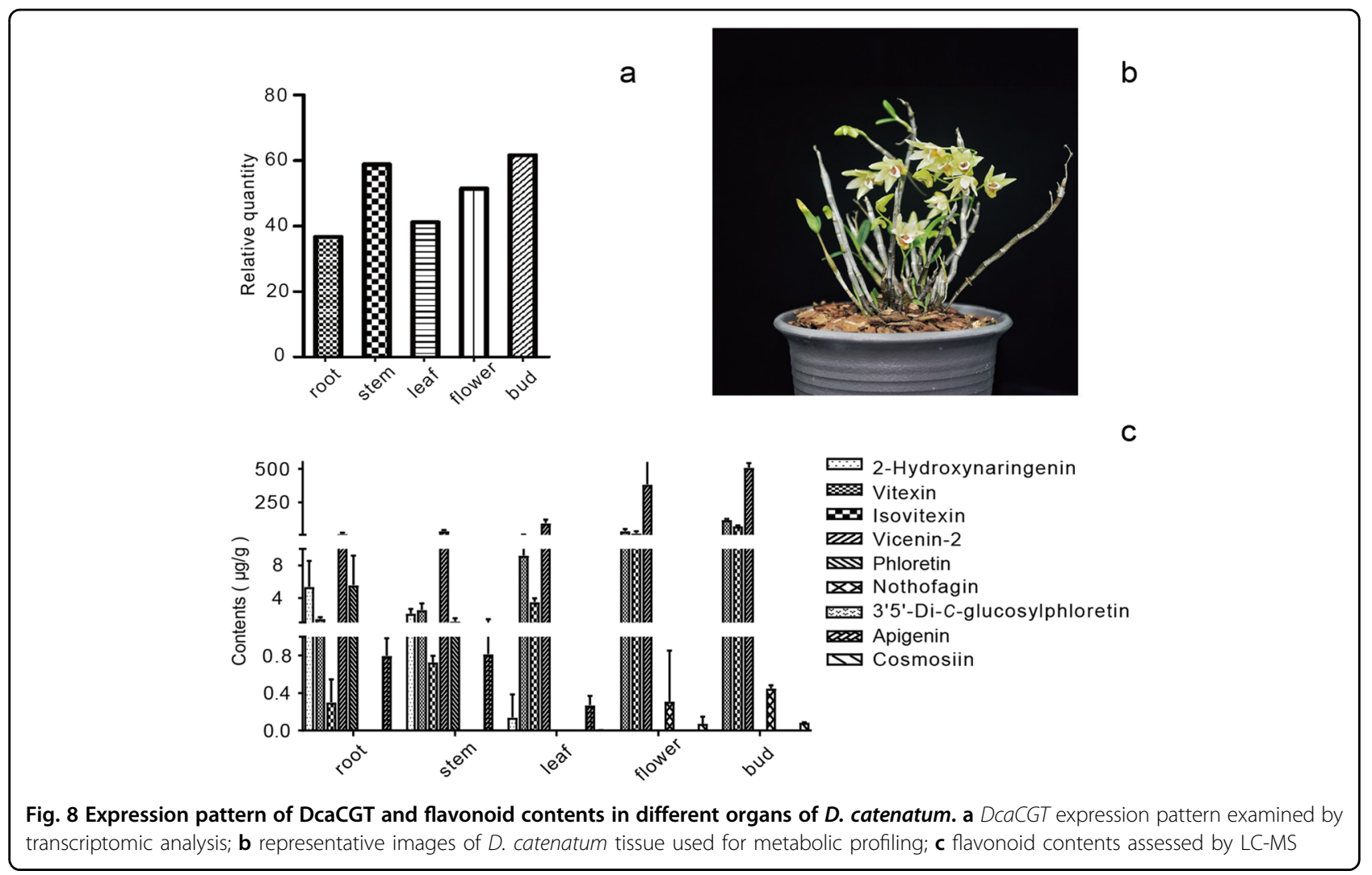

Table 1 Flavonoids in different organs of $D$. catenatum

\begin{tabular}{|c|c|c|c|c|c|c|c|c|}
\hline \multirow[t]{2}{*}{ Name } & \multirow[t]{2}{*}{$\mathrm{RT}(\min )$} & \multirow[t]{2}{*}{ Formula } & \multirow[t]{2}{*}{ Parent/product ion } & \multicolumn{5}{|c|}{ Flavonoid contents $(\bar{x} \pm s, \boldsymbol{\mu g} / \mathbf{g}, \boldsymbol{n}=\mathbf{3})$} \\
\hline & & & & Root & Srem & Leaf & Flower & Flower bud \\
\hline 2-Hydroxynaringenin & 8.59 & $\mathrm{C}_{15} \mathrm{H}_{12} \mathrm{O}_{6}$ & 286.97/151.00 & $5.39 \pm 3.17$ & $2.11 \pm 0.56$ & $0.14 \pm 0.24$ & n.d. & n.d. \\
\hline Vitexin & 5.81 & $\mathrm{C}_{21} \mathrm{H}_{20} \mathrm{O}_{10}$ & $430.95 / 311.00$ & $1.46 \pm 0.20$ & $2.55 \pm 0.80$ & $9.21 \pm 2.17$ & $38.88 \pm 13.69$ & $121.19 \pm 6.95$ \\
\hline Isovitexin & 6.03 & $\mathrm{C}_{21} \mathrm{H}_{20} \mathrm{O}_{10}$ & $430.88 / 311.00$ & $0.30 \pm 0.24$ & $0.73 \pm 0.07$ & $3.53 \pm 0.45$ & $21.36 \pm 15.36$ & $74.65 \pm 4.93$ \\
\hline Vicenin-2 & 3.64 & $\mathrm{C}_{27} \mathrm{H}_{30} \mathrm{O}_{15}$ & $592.99 / 352.90$ & $16.90 \pm 8.34$ & $38.61 \pm 6.97$ & $95.74 \pm 28.07$ & $383.52 \pm 187.65$ & $507.95 \pm 35.24$ \\
\hline Phloretin & 9.12 & $\mathrm{C}_{15} \mathrm{H}_{14} \mathrm{O}_{5}$ & $273.740 / 167.80$ & $5.59 \pm 3.60$ & $1.15 \pm 0.39$ & n.d. & n.d. & n.d. \\
\hline Nothofagin & 6.80 & $\mathrm{C}_{21} \mathrm{H}_{24} \mathrm{O}_{10}$ & $435.09 / 314.90$ & n.d. & n.d. & n.d. & $0.31 \pm 0.62$ & $0.45 \pm 0.03$ \\
\hline Apigenin & 9.26 & $\mathrm{C}_{15} \mathrm{H}_{10} \mathrm{O}_{5}$ & $268.76 / 117.10$ & $0.80 \pm 0.19$ & $0.82 \pm 0.61$ & $0.27 \pm 0.10$ & n.d. & n.d. \\
\hline Cosmosiin & 7.58 & $\mathrm{C}_{21} \mathrm{H}_{20} \mathrm{O}_{10}$ & $430.76 / 268.00$ & n.d. & n.d. & n.d. & $0.08 \pm 0.08$ & $0.09 \pm 0.00$ \\
\hline
\end{tabular}

n.d. not detected.

population pairwise Fst values among these clusters were higher than $0.25^{48}$. There was a greater degree of differentiation between Cluster_a and Cluster_c, with an Fstvalue of $0.58246(P<0.001)$, than between the other pairs of clusters. The differentiation between Cluster_a and Cluster_b and that between Cluster_b and Cluster_c were similar, with Fst values of $0.46405(P<0.001)$ and 0.47014 $(P<0.001)$, respectively.
Geographically, we found that the original Cluster_a was distributed only among 20 populations, mainly from Southwest, North, and easternmost China. Cluster_b covered 26 populations distributed in eastern and middle portions of China. Cluster_c, the newest evolutionary clade, contained all the populations except Xiushui, Jiangxi. Most populations in western China occupied a large proportion of Cluster_c (Fig. 10b). 


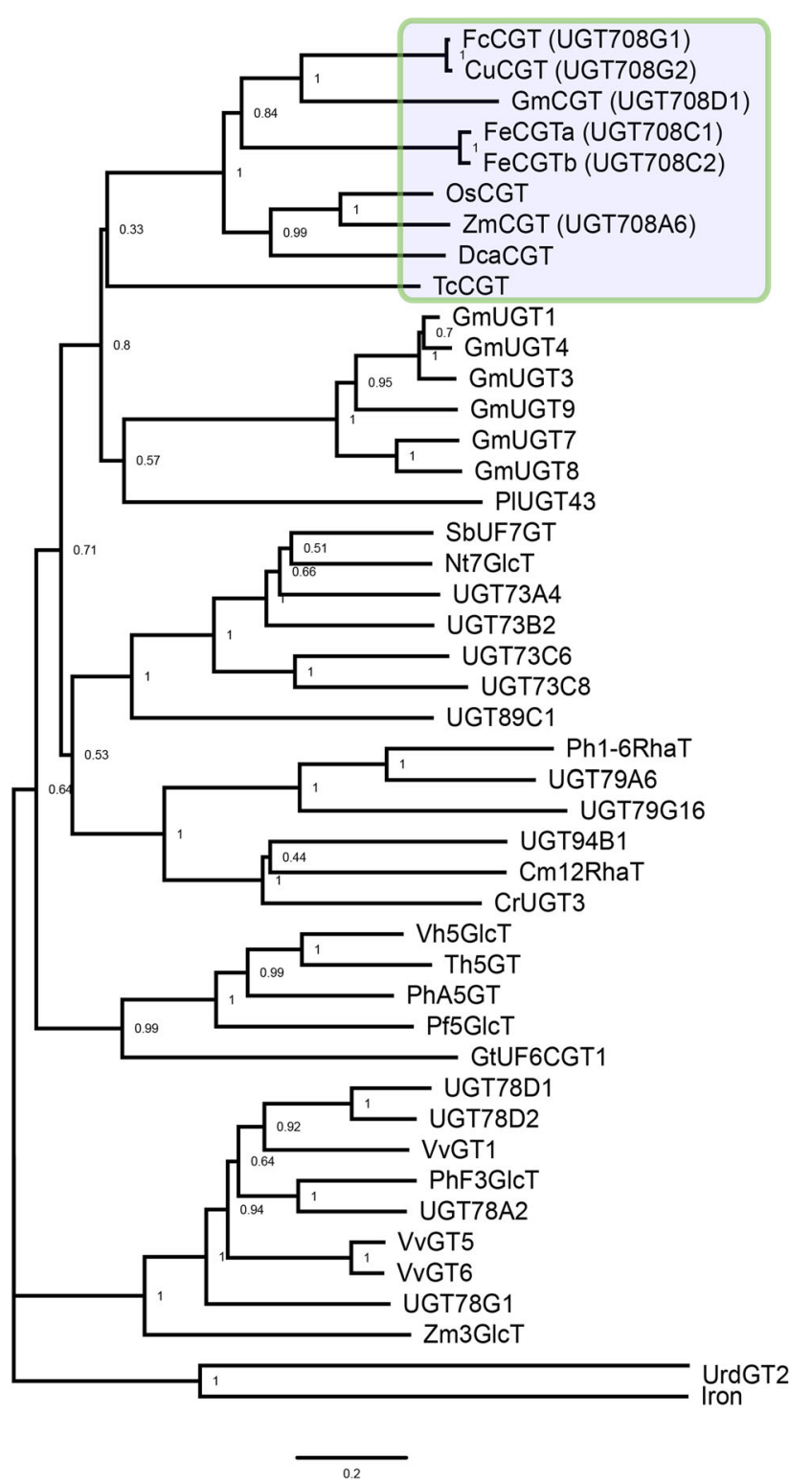

Cluster 6

Plant CGTs

Cluster 5

Isoflavone $7-\mathrm{OH}$

glycosylation / CGT

Cluster 1

Flavone $7-\mathrm{OH}$

glycosylation

Cluster 2

Cluster 4

$5-\mathrm{OH}$

glycosylation / CGT

Cluster 3

$3-\mathrm{OH}$

glycosylation

Cluster 7

Bacterial CGTs

Fig. 9 Neighbor-joining tree of DcaCGT and related glycosyltransferases. Phylogenetic analysis was performed by MEGA 7.0 using the neighbor-joining method and carried out via the bootstrapping method with 1000 replicates. The UGT amino-acid sequences were aligned using ClustalW. (Accession numbers are presented in Table S5)

To assess the genetic diversity of the populations, we calculated $\mathrm{Pi}$ and Tajima's $D$ values based on the distribution of 132 haplotypes among 34 populations. According to the direction of $D$. catenatum migration ${ }^{25}$, we found that the Tajima's $D$ value of the $D$. catenatum populations decreased from west to east, regardless of the presence or absence of ancestral populations. However, an upsurge in Pi from west to east was observed if the ancestral populations were removed (Fig. 10c, d). The diversity of eastern areas was higher than that of western areas after removing the ancestral populations (ancestral populations: Cluster_a/Cluster_abc $\geq 15 \%$ ). The Fst of the
34 populations ranged from 0 0.78766. Details of the molecular diversity, population pairwise Fst values, nucleotide diversity, and haplotype kinds and numbers are shown in Tables S6-8.

\section{Discussion}

In the present study, $82 U G T$ genes were identified in $D$. catenatum and clustered into 15 groups based on phylogenetic analysis. UGT gene products account for $\sim 0.28 \%$ of the gene products in $D$. catenatum, $<0.44 \%$ in A. thaliana, $0.36 \%$ in cotton, $0.4 \%$ in chickpea, and $>0.23 \%$ in maize ${ }^{17,18,21,49}$. In plants, 12 groups $(A-G, I-M)$ are 

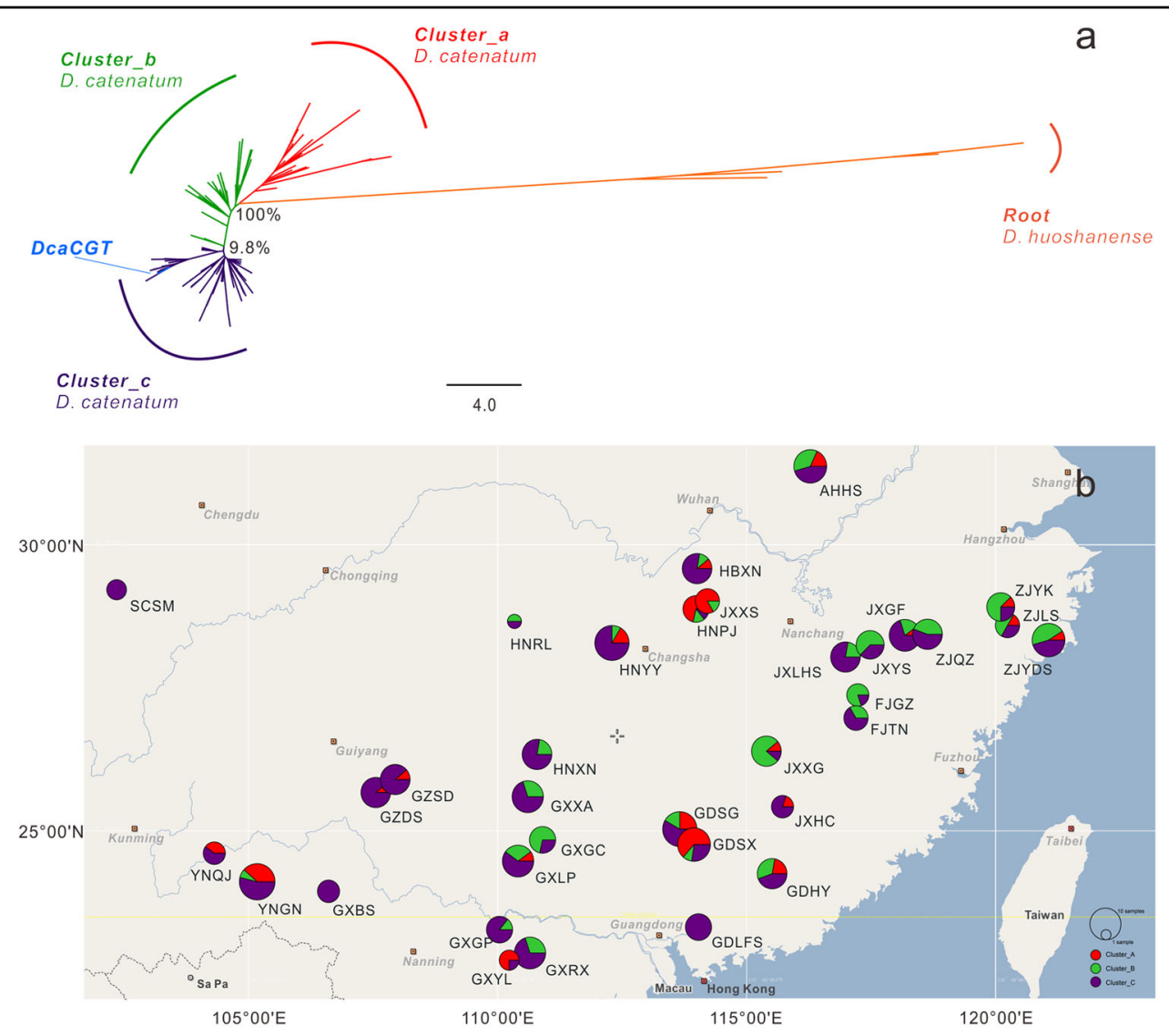

PI

C

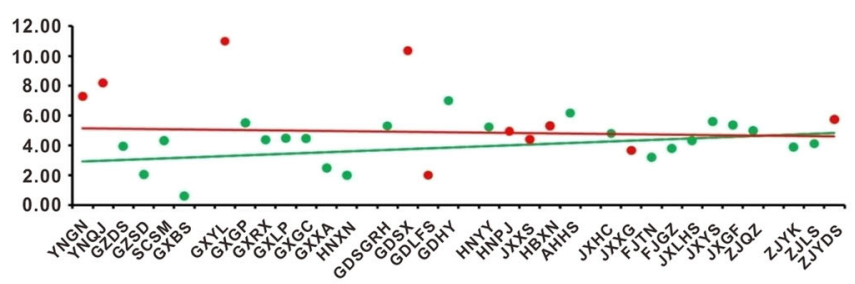

Tajima's $D$

d

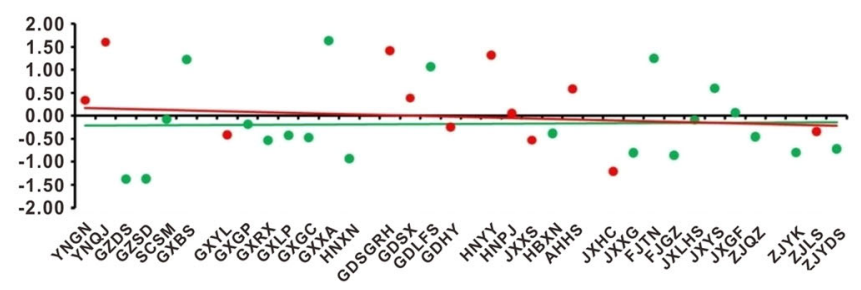

Fig. 10 Phylogenetic, geographic, and diversity analyses of DcaCGT in populations of $D$. catenatum. a Neighbor-joining tree. Bootstrap-values for the clusters are 100 and 9.8\%. b Geographic distribution of 274 D. catenatum accessions with three main clusters of 132 haplotypes. $\mathbf{c}$ Pi and $\mathbf{d}$ Tajima's D values of 33 populations. Red dots: ancestral populations; green dots: derived populations; red line: trendline with ancestral populations; green line: trendline without ancestral populations (ancestral populations: Cluster_a/Cluster_abc $\geq 15 \%$ )

highly conserved, with the exception of the $\mathrm{H}$ and $\mathrm{N}$ groups $^{18}$. Thus far, the absence of group $\mathrm{N}$ has been discovered in 22 species, only one of which is a monocot ${ }^{41}$. The loss of groups $\mathrm{N}$ and $\mathrm{H}$ during evolution suggests that either the functions of these groups are not important or these functions can be replaced by other 
factors ${ }^{19}$. Groups A, D, E, G, and L, which expanded more than others during the evolution of higher plants ${ }^{50}$, gained the highest, second highest, and third highest numbers (21 (D), 10 (E), and 8 (A, G, L)) members, accounting for $25 \%, 12 \%$, and $9.7 \%$ of the putative UGTs in D. catenatum, respectively. Members of these groups have been reported to catalyze a wide variety of substrates, such as flavonoids, terpenes, and alkaloids ${ }^{15,41}$, suggesting that $D$. catenatum is rich in these kinds of metabolites. Three groups, $\mathrm{O}, \mathrm{P}$, and $\mathrm{Q}$, which are found in maize but not $A$. thaliana, were also identified in $D$. catenatum. In accordance with a previous study, we concluded that group $\mathrm{O}$ in $D$. catenatum contained five closely related members, including four $Z O G$ genes $^{50}$. Members of group $\mathrm{O}$ may therefore catalyze the glycosylation of cytokinins ${ }^{18}$. Group Q, a group absent in Poales and Brassicales ${ }^{41}$, was also identified during our analysis. A novel group, R, which contains members of the UGT708 family, such as $Z m C G T$, was found in four members of $D$. catenatum. This suggests that there may be similar functional genes in $D$. catenatum.

Among the 82 genes that we identified, 78 UGTs were dispersed throughout the chromosomes. These UGTs were generally organized into clusters of 2-7 genes, and most ORFs in the clusters were oriented in the same direction. Genes in the same cluster showed high sequence similarity, reflecting their close phylogenetic relationships in the family and tandem duplication after orchid-wide genome duplication. This phenomenon also occurs in the GT1 family of cotton ${ }^{21}$. The position, phase, loss, and gain of introns can serve as important indicators for understanding the evolution of a gene family within phylogenetic groups. More than half (52\%) of UGTs lack introns, which is less than the number in maize $(60 \%)$ and Arabidopsis (58\%). Introns were abundantly present in phase 0 , suggesting that their phases remained stable. We also used the MEME web server to search the conserved motifs shared among UGT proteins. We found a total of 10 distinct conserved motifs, among which motif 1 , which encoded the UGT domain, was found in all the identified UGTs. From similar motif patterns, we concluded that differences among the groups were not significant and that the UGTs in D. catenatum are still undergoing evolution. Although most closely related members usually shared common motifs, some UGTs, such as Dca003346, Dca009408, and Dca014393, differed from the other members in their group. We speculated that the structural annotation of genes was incomplete. The specific motifs may contribute to the functional divergence of UGTs. Gene expression patterns can help us understand the function of these genes in different tissues. Thirty-five UGT genes were expressed among the nine tissues evaluated in this study. Active involvement of the abovementioned groups in each organ confirms that these groups have vital roles in growth hormone glycosylation. Groups D and E not only gained the most UGT members in $D$. catenatum but also possessed the most genes simultaneously expressed in all organs. Comparison of the day roots and night roots indicated that 12 UGTs were expressed either only during the day or only at night. This result suggests that some UGTs are expressed at specific times.

In recent years, genome-wide identification of gene families has been used to reveal pathways used to synthesize bioactive components such as geranyl and neryl glucoside in grape ${ }^{51}$ and 2-pheylethanol in peach ${ }^{22}$. In the present study, we compared the 82 Dca UGTs with identified CGTs using multiple sequence alignment and phylogenetic/evolutionary analysis. Putative genes, grouped into a clade with identified CGTs, were chosen as candidate genes for further verification. Based on enzyme activity, a putative di-C-glucosylflavone pathway in $D$. catenatum was proposed. Our results show that DcaCGT catalyzed both the first and second $C$-glycosylation reactions of di-C-glucosylflavone. This is the second enzyme possessing this catalytic ability, in addition to $\mathrm{FCCGT}^{38}$. DcaCGT showed strong activity when using phloretin as the substrate and weaker activity when using 2hydroxynaringenin as the substrate in vitro. When we used apigenin as a substrate, we found that a small amount of apigenin-7-O-glucoside remained in the reaction product. We concluded that DcaCGT not only catalyzed di-C-glucosylflavone but also processed partial active sites on $O$-glucosylflavone. However, we could not detect any $O$-glucosylflavone when we used phloretin or 2-hydroxynaringenin as a substrate. $C$-glucosylflavone was also not detected when using apigenin as a substrate. Thus, we deduced that DcaCGT exhibits high substrate specificity.

In our phytochemical analysis, the substrates 2-hydroxynaringenin, phloretin, and apigenin were detected only in the root, stem, and leaf, in which transcript expression was lower than that in the flowers and flower buds. The levels of the mono- $C$-glucosylflavonoid, di-C-glucosylflavonoid, and $O$-glucosylfavonoid products were higher in flower buds than in roots. Thus, we concluded that lower transcription led to a lower product content and that DcaCGT showed strong catalytic capacity in vivo. Comparing the amounts of the three products, we found that the accumulation of vicenin-2 and of its intermediate products vitexin and isovitexin was higher than that of nothofagin and cosmosiin in different tissues of $D$. catenatum. These results indicate that DcaCGT has a pivotal role in the accumulation of vicenin- 2 .

Flavonoids are used by organisms in response to nearly all abiotic stresses ${ }^{52,53}$. CGT, one of the key enzymes in the flavonoid biosynthesis pathway, has continued to expand during the evolution of $D$. catenatum. Our results 
show that Cluster_b/c demonstrated high differentiation resulting from ancestral and selection pressure, generating adaptable genotypes after $D$. catenatum migration. Based on the wide distribution of Cluster_c, we concluded that Cluster_c was the best genotype for acclimation. Interestingly, we found that DcaCGT was grouped into Cluster_c. Previous studies have reported that the accumulation of cosmosiin, which is a product of DcaCGT Oglycosylation, can strengthen the ability of $D$. catenatum to resist drought stress ${ }^{54,55}$. Most wild $D$. catenatum grows on cliffs with deficient water year round ${ }^{26}$, which may be the reason for the remaining $O$-glycosylation activity of DcaCGT. The distribution of Cluster_b led us to hypothesize that this genotype can better adapt to the environment of eastern and central China. However, whether CGTs in Cluster_b gained the same or other activities requires further study. Our results from population diversity analysis of $D$. catenatum migration show that populations in eastern China possess increased genetic variation. The ancestral Cluster_a can still be found in Yunnan, Guangdong, Zhejiang, and Hubei provinces. Yunnan and Guangdong are the ancestral region of D. catenatum $^{25}$, which is why it shows increased diversity. We concluded that human activity may lead to increased diversity in eastern areas of China. In addition, flavonoid contents in different $D$. catenatum organs indicate that multiple haplotypes may be one of the reasons for the different levels of vicenin-2 observed in different regions.

As the functions of plant CGTs have been increasingly identified, the reaction mechanisms of plant CGTs have also been gradually elucidated. Hirade et al. ${ }^{36}$ showed that substituting His 20 with alanine in UGT708D1 changes $\mathrm{C}$-glucosyltransferase activity to that of $\mathrm{O}$-glucosyltransferase; both D85 and R292 are active sites in C-glucosylation. Replacing either of these sites with alanine in UGT708D1 induces the loss of $C$-glucosylation. He et al. ${ }^{39}$ concluded that I94 and G284 are the two active sites for $C$-glucosylation. When I94 is replaced with glutamic acid or G284 is replaced with lysine, TcCGT changes from $C$ to $O$-glucosylation. Our haplotype analysis of DcaCGT from 34 populations indicated that the active sites of His and Asp among the 274 samples examined in this study were identical. However, the active site of lle was replaced by Met, and the active site of Gly was replaced by Arg. Such changes in active sites used for $C$-glucosylation may be the reason for $O$-glucosyltransferase activity. Further studies are needed to delineate the CGT reactions in detail.

In summary, we isolated a novel di-C-/O-glycosyltransferase from the $D$. catenatum GT1 family and analyzed the levels of related components in different tissues of $D$. catenatum. The results of transcriptomic and metabolic profiling demonstrated that the flavonoid distribution differed among organs. We further examined the origin and differentiation of DcaCGT and concluded that the remaining OGT activity may help $D$. catenatum adapt to water deficit stress. Based on previously identified active sites on UGT708D1 and TcCGT, we found that CGT active sites were altered in D. catenatum. However, we did not find distinct differences between the active sites of Cluster_b and Cluster_c. Our results show that changes in these active sites can produce OGT activity. However, further studies are needed to determine whether there are functional differences between Cluster_b and Cluster_c. Our study provides a basis for understanding the impact of environmental conditions on the biosynthesis of medically relevant compounds in $D$. catenatum. These results will lay the basis for enhancing vicenin-2 production using genetic strategies and molecular breeding in D. catenatum.

\section{Conclusion}

We performed a genome-wide analysis and functional verification of CGT genes in $D$. catenatum. The haplotype distribution of DcaCGT was assessed with 274 samples acquired from 34 populations. We isolated a novel $C$ glycosyltransferase (DcaCGT) from $D$. catenatum by identifying and analyzing 82 putative genes in the GT1 family. DcaCGT could specifically catalyze not only di-Cglycosylation but also $O$-glycosylation. Apart from the normal function of catalyzing 2-hydroxynaringenin and phloretin to the respective di- $C$-glycosides, DcaCGT also catalyzes apigenin to cosmosiin. We also established that DcaCGT functions expanded throughout the evolution of $D$. catenatum. The residual OGT activity may help $D$. catenatum resist drought stress. Our results reveal the biosynthetic pathways of $\mathrm{di}-\mathrm{C} / \mathrm{O}$-glucosylflavones in medicinal orchids, and our molecular phylogenetic analysis offers insight into the evolution of the functional gene.

\section{Materials and methods \\ Plant materials and chemicals}

D. catenatum was obtained from Qujing, Yunnan, and authenticated by Professor Zhongiian Liu (College of Landscape Architecture, Fujian Agriculture and Forestry University). Flower buds, flowers, leaves, stems, and roots, collected from healthy 2-year-old $D$. catenatum plants, were frozen at $-80^{\circ} \mathrm{C}$.

2-Hydroxynaringenin, vitexin, isovitexin, phloretin, apigenin, and cosmosiin were purchased from ChemFaces (Wuhan, China). UDP-glucose was purchased from Energy Chemical (Shanghai, China). Vicenin-2 was isolated from $D$. catenatum leaves in our laboratory. Nothofagin was isolated from Desmodium caudatum in Haifeng Chen's laboratory (College of Pharmacy, Xiamen University). 


\section{Identification of UGT genes}

To identify UGTs, a feature sequence of UGTs, the 44amino-acid conserved sequence of the PSPG motif, was used as a query to perform a local BLASTP search against the $D$. catenatum genome database. The coding sequence and chromosome distribution were obtained from the same database. The molecular weight (MW) and isoelectric point (pI) of each UGT protein were calculated using the online ExPASy (http://web.expasy.org/ compute_pi/) program. The domain of each UGT protein was obtained using an NCBI batch CD search (https://www.ncbi.nlm.nih.gov/cdd/). KofamKOALA (https://www.genome.jp/tools/kofamkoala/) was used to confirm the function of UDP-glycosyltransferase among the UGT candidates. The subcellular localization of each UGT protein was predicted using the online CELLO v2.5 system (http://cello.life.nctu.edu.tw/). The distribution of UGT genes and their location on chromosomes were visualized using TB tools (https://omictools.com/ tbtools-tool).

\section{Sequence alignment and phylogenetic analysis}

The predicted amino-acid sequences of $D$. catenatum UGTs were aligned by the neighbor-joining method in ClustalX software using 10,000 bootstrap replicates. A phylogenetic tree was constructed using FigTree 1.4.3.

\section{Sequence analysis}

The exon-intron organization of D. catenatum UGTs was evaluated by determining intron lengths, positions, and phases in the genome. Intron phases were determined as follows: introns positioned between two triplet codons were defined as phase 0 ; introns positioned after the first and second base in the codon were defined as phases 1 and 2, respectively. Conserved motifs were analyzed using the MEME online program (http://meme.nbcr.net/meme/ intro.html).

\section{Gene expression analysis using RNA-seq}

Transcriptomic data available for putative $U G T$ expression in different plant organs were analyzed according to the data published by Zhang et al. ${ }^{29}$. Samples of each organ (flower bud, gynandrium, labellum, sepal, root tip, root, leaf, stem, and pollen), obtained from three or more different plants, were pooled prior to sequencing. Gene expression levels are shown as FPKM values. Transcript profiles for selected D. catenatum UGTs were expressed as heatmaps.

\section{Cloning of DcaCGT and production of recombinant protein}

Total RNA of $D$. catenatum was extracted from frozen leaves using a Quick RNA Isolation Kit per the manufacturer's instructions (Huayueyang, Beijing, China). Firststrand cDNA was synthesized from total RNA (568 ng) using TransScript All-in-One First-Strand cDNA Synthesis SuperMix for PCR (TransGen Biotech, Beijing, China). PCR was performed using $1 \mu \mathrm{L}$ first-strand cDNA as a template and DcaCGT-F and DcaCGT-R as primers (the primer sequences are provided in Table S3). The coding sequence (CDS) of the candidate CGT gene, DcaCGT, was amplified using PrimerSTAR Max DNA polymerase (Takara Bio, Japan) under the following conditions: $94{ }^{\circ} \mathrm{C}$ for $5 \mathrm{~min}, 37$ cycles of $94{ }^{\circ} \mathrm{C}$ for $30 \mathrm{sec}, 55^{\circ} \mathrm{C}$ for $30 \mathrm{sec}$, and $72{ }^{\circ} \mathrm{C}$ for $90 \mathrm{sec}$, followed by $72^{\circ} \mathrm{C}$ for $10 \mathrm{~min}$. The amplified fragment was purified from an agarose gel (Magen, China), cloned using pEAST-T5 vector (TransGen Biotech, Beijing, China), transformed in phage-resistant chemically competent $E$. coli cells (TransGen Biotech, Beijing, China), and sequenced.

The empty vector pET32a $(+)$ (Novagen, USA) was digested with FastDigest enzymes Sca I and EcoR V (Thermo, USA). Full-length cDNA was amplified by PCR using pETF-DcaCGT and pETR-DcaCGT as primers (Table S3) and purified from an agarose gel. To obtain the recombinant plasmid, the target gene was linked with a linearized vector using an Infusion cloning system (Clontech, Japan) and transformed into E. coli Transetta (DE3) (TransGen Biotech, Beijing, China); $0.01 \mathrm{~mm}$ isopropyl $\beta$ - $\mathrm{d}$-1-thiogalactopyranoside was then added, and the cells were incubated for $20 \mathrm{~h}$ at $16^{\circ} \mathrm{C}$. The cell cultures were centrifuged at $6574 \times g$, after which the supernatants were removed and harvested $E$. coli cells were stored at $-20^{\circ} \mathrm{C}$. The $E$. coli cells were suspended in $1 \times$ PBS (containing $1 \mathrm{~mm}$ PMSF and $20 \mathrm{~mm}$ imidazole) and disrupted by sonication. After centrifugation at $15,000 \times g$ for $30 \mathrm{~min}$ at $4{ }^{\circ} \mathrm{C}$, the supernatants were applied to a Ni-NTA Superflow column (Qiagen, Germany) with a linear gradient of imidazole, followed by desalination-exchange buffer ( $\mathrm{pH}$ 8.0) containing $1 \mathrm{~mm}$ DTT on a PD-10 Column (GE Healthcare).

\section{UGT enzymatic activity assay}

Enzymatic reactions were performed at $30^{\circ} \mathrm{C}$ for 5-90 min using a $250 \mu \mathrm{m}$ acceptor substrate, UDP-glucoside, $1 \mathrm{~mm}$ DTT, $50 \mathrm{~mm}$ Tris- $\mathrm{HCl}$ (pH 8.0), and $300 \mathrm{ng}$ purified protein, resulting in a total volume of $100 \mu \mathrm{l}$. The reaction was terminated by adding $10 \mu \mathrm{L} 2 \mathrm{M} \mathrm{HCl}$, followed by incubation at $60^{\circ} \mathrm{C}$ for $30 \mathrm{~min}$ to dehydration. The protein was boiled at $100^{\circ} \mathrm{C}$ for $10 \mathrm{~min}$ as a negative control.

Supernatants were dissolved in $100 \mu \mathrm{l} \mathrm{HPLC-grade}$ methanol and analyzed via HPLC-MS/MS on an AB Sciex Triple-TOF $5600^{+}$mass spectrometer coupled to a Shimadzu LC-30 AD chromatography system. Products were detected by measuring absorbance at $280 \mathrm{~nm}$ and $340 \mathrm{~nm}$.

\section{HPLC-MS analysis}

Flower buds, flowers, leaves, stems, and roots of $D$. catenatum were ground into a powder in liquid nitrogen. 
In total, $100 \mathrm{mg}$ powder was weighed and transferred into a conical flask. Then, $40 \mathrm{~mL}$ methanol was added, and the mixture was extracted ultrasonically for $1 \mathrm{~h}$ at $30^{\circ} \mathrm{C}$. Extracts were concentrated using a hypobaric drying method at $60^{\circ} \mathrm{C}$. Then, $5 \mathrm{~mL}$ HPLC-grade methanol was used to redissolve the residues to obtain the extraction solution. All the samples were filtered through a $0.22-\mu \mathrm{m}$ membrane filter before analysis via HPLC-MS. 2-Hydroxynaringenin, vitexin, isovitexin, vicenin-2, phloretin, nothofagin, apigenin, and cosmosiin were confirmed by HPLC-MS analysis using authentic standards. 3', 5'-Di-Cglucosylphloretin was identified by the $\mathrm{MS}^{2}$ fragment. Loratadine was chosen as the internal standard. The quantification of these flavonoids was performed based on their corresponding standard curves. Each tissue type was analyzed in three biological replicates in this experiment.

HPLC-MS analysis was performed with UPLC-MS/MS (AB Sciex Triple-TOF $5600^{+}$mass spectrometer coupled to a Shimadzu LC-30 AD chromatography system) to complete qualitative analysis. The detected components were sent to a Thermo Fisher UHPLC-MS/MS system consisting of an Accela ultra-high-pressure liquid chromatograph and a TSQ Quantum Ultra triple-quadrupole mass spectrometer (Thermo Fisher Scientific, San Jose, CA, USA) fitted with an ESI source for quantitative analysis. All separations were carried out with an SB-C18 column $(2.1 \times 100 \mathrm{~mm}, 1.8 \mu \mathrm{m}$; Agilent $)$. The system was operated in negative ion mode at a flow rate of $0.3 \mathrm{~mL} /$ min using solvent $\mathrm{A}$ (water with $0.1 \%$ formic acid), solvent $\mathrm{B}$ (acetonitrile), and $\mathrm{C}$ (methanol). The gradient elution started from 7\% B, 7\% C for 0 min, followed by $9 \%$ B, 9\% C in $0.3 \mathrm{~min} ; 11 \% \mathrm{~B}, 11 \% \mathrm{C}$ in $2.7 \mathrm{~min} ; 13 \% \mathrm{~B}, 13 \% \mathrm{C}$ in $3.8 \mathrm{~min} ; 14 \% \mathrm{~B}, 14 \% \mathrm{C}$ in $4.8 \mathrm{~min} ; 18 \% \mathrm{~B}, 18 \% \mathrm{C}$ in $6.5 \mathrm{~min} ; 45 \% \mathrm{~B}, 45 \% \mathrm{C}$ in $8.5 \mathrm{~min}$; a hold for $0.7 \mathrm{~min}$; and a post run time of $1.5 \mathrm{~min}$ for re-equilibration. An optimal MS signal response of eight analytes and IS was obtained with the following ion source parameters: spray voltage at $3500 \mathrm{~V}$, capillary temperature at $320^{\circ} \mathrm{C}$, sheath gas pressure at $35 \mathrm{psi}$, and auxiliary gas pressure at $10 \mathrm{psi}$.

\section{Haplotype analysis of DcaCGT}

A total of 274 samples of D. catenatum from 34 populations and five samples of $D$. huoshanense from one population were newly resequenced using 150 paired-end cycles on a NovaSeq 6000 instrument. After quality trimming, paired-end reads were mapped to the $D$. catenatum reference genome using BWA-MEM v0.7.12r1039 with default parameters ${ }^{56}$. The SortSam and MarkDuplicates commands from Picard Tools v1.56 were used to mark and remove duplicates in the bam-format files. IndelRealigner and RealignerTargetCreator from GenomeAnalysisTK v3.8-1 were used to perform local realignment ${ }^{57}$. For each sample, individual variant calling was conducted using Haplotyper and GVCFtyper from
Sentieon v201711.03 ${ }^{58}$. Coding sequences for DcaCGT from 274 samples were obtained using the faidx tool from SAMtools v1.9 and consensus from BCFtools v1.2 ${ }^{59}$.

The 274 DcaCGT sequences were aligned by ClustalX software in MEGA5. A phylogenetic tree was constructed using the DcaCGT-coding sequences and neighborjoining method in MEGA5. Population genetic analysis was performed by using DnaSP $6{ }^{60}$. Nei's nucleotide diversity $(\pi)$ and Fst were computed by Arlequin v.3.0 $0^{61}$.

\begin{abstract}
Acknowledgements
We are very thankful to Haifeng Chen's team (Xiamen University) for providing the necessary standard and Shaofeng Wu (Guangzhou University of Chinese Medicine) for providing the laboratory to accomplish our work. This project was supported by the 2018 Shaoguan City Science and Technology Plan Project: Special Project of Industry-University-Research Cooperation (no. 2018CS11919), 2019 Guangdong Province Special Fund for Science and Technology ('Big project + task list') Project: Ecological Cultivation and Sustainable Utilization of Danxia Dendrobium officinale, a rare Southern Medicine in Guangdong Province (no. 2019gdskjzxzj-zt3-2), Fundamental Research Project of Shenzhen, China (JCYJ20170817105300166), National Key Research and Development Program of China (no. 2018YFD1000401) and Key Laboratory of National Forestry and Grassland Administration for Orchid Conservation and Utilization Construction Funds (nos. 115/118990050;115/ KJG18016A) awarded to Z.-J.L.
\end{abstract}

\section{Author details}

${ }^{1}$ School of Pharmaceutical Sciences, Guangzhou University of Chinese Medicine, Guangzhou 510006, China. ${ }^{2}$ Shantou University Medical College, Shantou 515041, China. ${ }^{3}$ State Key Laboratory of Systematic and Evolutionary Botany, Institute of Botany, Chinese Academy of Sciences, Beijing 100093, China. ${ }^{4}$ University of Chinese Academy of Sciences, Beijing 100049, China. ${ }^{5}$ Shenzhen Key Laboratory for Orchid Conservation and Utilization, The National Orchid Conservation Center of China and The Orchid Conservation and Research Center of Shenzhen, Shenzhen 518114, China. ${ }^{6}$ Department of Pharmacy, Sun Yat-Sen Memorial Hospital, Sun Yat-Sen University, Guangzhou 510120 Guangdong, China. ${ }^{7}$ Shaoguan Institute of Danxia Dendrobium Officinale, Shaoguan 512005, China. ${ }^{8}$ Key Laboratory of National Forestry and Grassland Administration for Orchid Conservation and Utilization, College of Landscape Architecture, Fujian Agriculture and Forestry University, Fuzhou 350002, China. ${ }^{9}$ Henry Fok College of Biology and Agriculture, Shaoguan University, Shaoguan 512005, China

\section{Author contributions}

Z.R. and X.J. carried out the discovery and functional identification of DcaCGT and wrote this manuscript. Z.J. and G.Z. analyzed the population diversity. Y.L., S.T., and J.Z. carried out the LC-MS experiments. Z.L. and Y.W. collected the samples and extracted DNA. G.W and Z.L. are the corresponding authors and contributed significantly to the work design.

Conflict of interest

The authors declare that they have no conflict of interest.

Supplementary Information accompanies this paper at (https://doi.org/ 10.1038/s41438-020-0330-4).

Received: 6 February 2020 Revised: 20 April 2020 Accepted: 28 April 2020 Published online: 01 July 2020

\footnotetext{
References

1. Ulrike, M. Flavonoid functions in plants and their interactions with other organisms. Plants 7, 30 (2018).

2. Casati, P. \& WALBOT, V. Differential accumulation of maysin and rhamnosylisoorientin in leaves of high-altitude landraces of maize after UV-B exposure. Plant Cell Environ. 28, 788-799 (2005).
} 
3. Mommer, L., Kirkegaard, J. \& van Ruijven, J. Root-root interactions: towards a rhizosphere framework. Trends Plant Sci. 21, 209-217 (2016).

4. Hooper, A. M. et al. Isoschaftoside, a C-glycosylflavonoid from Desmodium uncinatum root exudate, is an allelochemical against the development of Striga. Phytochemistry 71, 904-908 (2010).

5. Yabuya, T., Nakamura, M., Iwashina, T., Yamaguchi, M. \& Takehara, T. Anthocyanin-flavone copigmentation in bluish purple flowers of Japanese garden iris (Iris ensata Thunb.). Euphytica 98, 163-167 (1997).

6. Marrassini, C., Davicino, R., Acevedo, C., Anesini, C. \& Ferraro, G. Vicenin-2, a potential anti-inflammatory constituent of Urtica circularis. J. Nat. Prod. 74, 1503-1507 (2011)

7. Islam, M. N., Ishita, I. J., Jung, H. A. \& Choi, J. S. Vicenin 2 isolated from Artemisia capillaris exhibited potent anti-glycation properties. Food Chem. Toxicol. 69, 55-62 (2014).

8. Verspohl, E. J., Fujii, H., Homma, K. \& Buchwald-Werner, S. Testing of Perilla frutescens extract and Vicenin 2 for their antispasmodic effect. Phytomedicine 20, 427-431 (2013).

9. Lee, W., Yoon, E. K., Kim, K. M., Dong, H. P. \& Bae, J. S. Anti-septic effect of vicenin-2 and scolymoside from Cyclopia subternata (Honeybush) in response to HMGB1 as a late sepsis mediator in vitro and in vivo. Can. J. Physiol. Pharm. 93, 709 (2015).

10. Lee, W. \& Bae, J.-S. Antithrombotic and antiplatelet activities of vicenin-2. Blood Coagul. Fibrinolysis 26, 628-634 (2015).

11. Luo, Y. et al. Structure identification of viceninii extracted from and the reversal of tgf- $\beta 1$-induced epithelial'mesenchymal transition in lung adenocarcinoma cells through TGF- $\beta /$ Smad and PI3K/Akt/mTOR signaling pathways. Molecules 24, 144 (2019).

12. Nagaprashantha, L. D. et al. Anti-cancer effects of novel flavonoid vicenin-2 as a single agent and in synergistic combination with docetaxel in prostate cancer. Biochem. Pharmacol. 82, 1100-1109 (2011).

13. Li, P., Lei, K., Li, Y., He, X. \& Hou, B. Identification and characterization of the first cytokinin glycosyltransferase from rice. Rice 12, 19-30 (2019).

14. Stevenson, P. C., Nicolson, S. W. \& Wright, G. A. Plant secondary metabolites in nectar: impacts on pollinators and ecological functions. Funct. Ecol. 31, 65-75 (2017).

15. Brazier-Hicks, M., Gershater, M., Dixon, D. \& Edwards, R. Substrate specificity and safener-inducibility of the plant UDP-glucose dependent family 1 glycosyltransferases super-family. Plant Biotechnol. J. 16, 337-348 (2018).

16. Peng, $M$. et al. Differentially evolved glucosyltransferases determine natural variation of rice flavone accumulation and UV-tolerance. Nat. Commun. 8 , 1975 (2017).

17. Li, Y., Baldauf, S., Lim, E.-K. \& Bowles, D. J. Phylogenetic analysis of the UDPglycosyltransferase multigene family of Arabidopsis thaliana. J. Biol. Chem. 276, 4338-4343 (2001)

18. Li, Y. et al. Genome-wide identifcation and phylogenetic analysis of Family-1 UDP glycosyltransferases in maize (Zea mays). Planta 239, 1265-1279 (2014).

19. He, Y. et al. Genome-wide analysis of family-1 UDP glycosyltransferases (UGT) and identification of UGT genes for FHB resistance in wheat (Triticum aestivum L.). BMC Plant Biol. 18,67 (2018).

20. Mamoon Rehman, $\mathrm{H}$. et al. Genome-wide analysis of family-1 UDP-glycosyltransferases in soybean confirms their abundance and varied expression during seed development. J. Plant Physiol. 206, 87-97 (2016).

21. Huang, J. et al. Genome-wide analysis of the family 1 glycosyltransferases in cotton. Mol. Genet. Genomics 290, 1805-1818 (2015).

22. Wu, B. et al. Genome-wide identification, expression patterns, and functional analysis of UDP glycosyltransferase family in peach (Prunus persica L. Batsch). Front. Plant Sci. 8, 389 (2017)

23. Cheng, $\mathbf{X}$. et al. Family-1 UDP glycosyltransferases in pear (Pyrus bretschneideri): molecular identification, phylogenomic characterization and expression profiling during stone cell formation. Mol. Biol. Rep. 46, 2153-2175 (2019).

24. Zhang, Y. et al. Dendrobium officinale leaves as a new antioxidant source. $J$. Funct. Foods 37, 400-415 (2017).

25. Hou, B. et al. Iteration expansion and regional evolution: phylogeography of Dendrobium officinale and four related taxa in southern China. Sci. Rep. 7, 43525 (2017).

26. Lei, Z. et al. Transcriptome analysis reveals genes involved in flavonoid biosynthesis and accumulation in dendrobium catenatum from different locations. Sci. Rep. 8, 6373 (2018)

27. Teixeira, dS., Jaime, A. \& Ng, T. B. The medicinal and pharmaceutical importance of Dendrobium species. Appl. Microbiol. Biot. 101, 2227-2239 (2017).
28. Yuan, Y. et al. Tissue-specific transcriptome for Dendrobium officinale reveals genes involved in flavonoid biosynthesis. Genomics 112, 1781-1794 (2020).

29. Zhang, G. Q. et al. The Dendrobium catenatum Lindl. genome sequence provides insights into polysaccharide synthase, floral development and adaptive evolution. Sci. Rep. 6, 1-10 (2016).

30. Zhang, $X$. et al. Identifcation and quantitative analysis of phenolic glycosides with antioxidant activity in methanolic extract of Dendrobium catenatum flowers and selection of quality control herb-markers. Food Res. Int. 123, 732-745 (2019).

31. Wang, K. et al. Dendrobium officinale polysaccharide attenuates type 2 diabetes mellitus via the regulation of PI3K/Akt-mediated glycogen synthesis and glucose metabolism. J. Funct. Foods 40, 261-271 (2018).

32. $\mathrm{Yu}, \mathrm{S}$. et al. Gigantol inhibits Wnt/ß-catenin signaling and exhibits anticance activity in breast cancer cells. BMC Complement. Altern. Med. 18, 59 (2018).

33. Brazier-Hicks, M. et al. The C-glycosylation of flavonoids in cereals. J. Biol. Chem. 284, 17926-17934 (2009).

34. Falcone Ferreyra, M. L. et al. Identification of a bifunctional maize C- and Oglucosyltransferase. J. Biol. Chem. 288, 31678-31688 (2013).

35. Nagatomo, Y. et al. Purification, molecular cloning and functional characterization of flavonoid C-glucosyltransferases from buckwheat (Fagopyrum esculentum) cotyledon. Plant J. 80, 437-448 (2014).

36. Hirade, $Y$. et al. Identification and functional analysis of 2-hydroxyflavanone Cglucosyltransferase in soybean (Glycine max). FEBS Lett. 589, 1778-1786 (2015).

37. Sasaki, N. et al. Identification of the glucosyltransferase that mediates direct flavone C-glucosylation in Gentiana triflora. FEBS Lett. 589, 182-187 (2014).

38. Ito, T., Fujimoto, S., Suito, F., Shimosaka, M. \& Taguchi, G. C-Glycosyltransferases catalyzing the formation of di-C-glucosyl flavonoids in citrus plants. Plant J. 91, 187-198 (2017).

39. He, J.-B. et al. Molecular characterization and structural basis of a promiscuous Cglycosyltransferase from Trollius chinensis. Angew. Chem. 58, 11513-11520 (2019).

40. $\mathrm{Ng}, \mathrm{T}$. B. et al. Review of research onDendrobium, a prized folk medicine. Appl. Microbiol. Biot. 93, 1795-1803 (2012).

41. Wilson, A. \& Tian, L. Phylogenomic analysis of UDP-dependent glycosyltransferases provides insights into the evolutionary landscape of glycosylation in plant metabolism. Plant J. 100, 1273-1288 (2019).

42. Zhang, G. Q. et al. The Apostasia genome and the evolution of orchids. Nature 549, 379-383 (2017).

43. Pereira, C. A. M., Yariwake, J. H. \& Mccullagh, M. Distinction of the Cglycosylfavone isomer pairs orientin/isoorientin and vitexin/isovitexin using HPLC-MS exact mass measurement and in-source CID. Phytochem. Anal. 16, 295-301 (2005).

44. Silva, D. B. et al. Mass Spectrometry of Flavonoid Vicenin-2, Based Sunlight Barriers in Lychnophora species. Sci. Rep. 4, 4309 (2014).

45. Stalmach, A. L., Mullen, W., Pecorari, M., Serafini, M. \& Crozier, A. Bioavailability of C-linked dihydrochalcone and flavanone glucosides in humans following ingestion of unfermented and fermented rooibos teas. J. Agric. Food Chem. 57, 7104-7111 (2009)

46. Roowi, S. \& Crozier, A. Flavonoids in tropical citrus species. J. Agric. Food Chem. 59, 12217-12225 (2011)

47. March, R. E., Lewars, E. G., Stadey, C. J., Miao, X. S. \& Metcalfe, C. D. A comparison of flavonoid glycosides by electrospray tandem mass spectrometry. Int. J. Mass Spectrom. 248, 61-85 (2006).

48. Wright, S. The interpretation of population structure by F-statistics with special regard to systems of mating. Evolution 19, 395-420 (1965).

49. Ranu, S., Vimal, R., G, S. C. \& Sara, A. Genome-wide identification and tissuespecific expression analysis of udp-glycosyltransferases genes confirm their abundance in Cicer arietinum (chickpea) genome. Plos ONE 9, e109715 (2014).

50. Caputi, L., Malnoy, M., Goremykin, V., Nikiforova, S. \& Martens, S. A genomewide phylogenetic reconstruction of family 1 UDP-glycosyltransferases revealed the expansion of the family during the adaptation of plants to life on land. Plant J. 69, 1030-1042 (2011).

51. Bönisch, F. et al. Activity-based profiling of a physiologic aglycone library reveals sugar acceptor promiscuity of family 1 udp-glucosyltransferases from grape. Plant Physiol. 166, 23-39 (2014).

52. Nakabayashi, R. \& Saito, K. Integrated metabolomics for abiotic stress responses in plants. Curr. Opin. Plant Biol. 24, 10-16 (2015).

53. Nan et al. Malus sieversii: the origin, flavonoid synthesis mechanism, and breeding of red-skinned and red-fleshed apples. Hortic. Res. 5, 70 (2018).

54. Mechri, B. et al. Verbascoside and oleuropein are potential indicators of drought resistance in olive trees (Olea europaea L.). Plant. Physiol. Biochem. 141, 407-414 (2019). 
55. Hojati, M., Modarres-Sanavy, S. A. M., Ghanati, F. \& Panahi, M. Hexaconazole induces antioxidant protection and apigenin-7-glucoside accumulation in Matricaria chamomilla plants subjected to drought stress. J. Plant Physiol. 168, 782-791 (2011).

56. Li, H. \& Durbin, R. Fast and accurate short read alignment with BurrowsWheeler transform. Bioinformatics 25, 1754-1760 (2009).

57. Mckenna, A. et al. The Genome Analysis Toolkit: a MapReduce framework for analyzing next-generation DNA sequencing data. Genome Res. 20, 1297-1303 (2010).
58. Freed, D. N., Aldana, R., Weber, J. A. \& Edwards, J. S. The Sentieon Genomics Tools - a fast and accurate solution to variant calling from next-generation sequence data. bioRxiv 115717 (2017).

59. Danecek, P. \& McCarthy, S. A. BCFtools/csq: haplotype-aware variant consequences. Bioinformatics 33, 2037-2039 (2017).

60. Librado, P. \& Rozas, J. DnaSP v5: a software for comprehensive analysis of DNA polymorphism data. Bioinformatics 25, 1451-1452 (2009).

61. Laurent et al. Arlequin (version 3.0): an integrated software package for population genetics data analysis. Evol. Bioinform. Online 1, 47-50 (2007). 